\title{
DECONFINEMENT IN QCD WITH DYNAMICAL QUARKS \&
}

\author{
O. Borisenko, M. Faber \\ Institut für Kernphysik, Technische Universität Wien, \\ Wiedner Hauptstr. 8-10, A-1040 Vienna, Austria \\ and G. Zinovjev \\ Institute for Theoretical Physics, Ukrainian Academy of Science, Kiev 143
}

\begin{abstract}
We study the phase structure of full QCD within the canonical ensemble (CE) with respect to triality in a lattice formulation. The procedure for the calculation of the effective potentials (EP) in the CE is given. We calculate the EP for the three dimensional $S U(2)$ gauge model at finite temperatures in the strong coupling region. The potential exhibits a genuine deconfinement phase transition unlike the similar potential obtained in the grand canonical ensemble (GCE) which demonstrates explicit $Z\left(N_{c}\right)$ symmetry breaking at any temperature. Furthermore, we investigate the EP with the chiral condensate included. In contradiction to other authors, we find chiral symmetry restoration in all triality sectors. In the scheme with massless staggered fermions we observe chiral symmetry restoration accompanying a deconfinement phase transition of first order. Above the critical point, besides two $Z(2)$ symmetric "deconfining" vacua there exists a metastable "confining" vacuum in a wide region of the $\left(N_{t}, \gamma\right)$-plane. Such a picture could be interpreted as an indication on a mixed state of hadrons and quarks in the vicinity of the critical line.
\end{abstract}

\footnotetext{
${ }^{1}$ Supported by Bundesministerium für Wissenschaft, Forschung und Kunst of Austria
} 
A comprehensive understanding of the phase structure of Quantum Chromodynamics (QCD) is the basis for many phenomenological applications, for possible signals of the quark-gluon plasma and other topics (see, for instance, the review on the Symposium 'Lattice-94' [1]). In numerical calculations a number of new results has been obtained. Nevertheless, the theoretical understanding of the phase structure of full QCD, despite huge efforts, is almost on the same level as it has been around fifteen years ago. In our view, one of the central points of the problem is a reliable theoretical determination of the deconfinement phase transition in QCD with dynamical fermions. It was understood in the beginning of eighties [2] that in pure gluodynamics deconfinement can be described in terms of the spontaneous breakdown of the global $Z\left(N_{c}\right)$ symmetry of the vacuum. An appropriate order parameter, the Polyakov loop, was proposed to distinguish the confined phase of static colour charges from the deconfinement. Since loops of dynamical quarks violate in general the $Z\left(N_{c}\right)$ symmetry explicitly, a naive application of this picture to full QCD is not possible. At large values of quark masses a reminiscent of deconfinement can be seen in the behaviour of some thermodynamical functions. In QCD with massless quarks the behaviour of the quark condensate demonstrates a phase transition associated with the restoration of chiral symmetry. It was stressed, however, that "with two flavors of light quarks in the staggered fermion scheme, there appears to be a dramatic crossover, but so far no evidence for a genuine phase transition" [1] (see also [3] for review of the thermal transition in QCD).

Recently, in several articles [4, 5, 6] strong arguments have been given that the general statement "dynamical quarks in QCD breaks $Z\left(N_{c}\right)$ symmetry explicitly", is not valid. It has been discussed that the fermionic determinant in finite temperature QCD describes a grand canonical ensemble (GCE). One can restrict the statistical averaging procedure to a canonical ensemble (CE) with $n$-ality zero which may still be the grand canonical with respect to baryon number. This CE does not break $Z\left(N_{c}\right)$ symmetry explicitly.

In Ref.[5] we speculated about the possible QCD phase structure. We gave some arguments that there should be a genuine deconfinement phase transition while in the GCE only a crossover has been observed. Here, we would like to supplement our intuitive arguments by a calculation of the effective potential in the $\mathrm{CE}$ at strong coupling. Since the $Z\left(N_{c}\right)$ symmetry is not explicitly broken in this ensemble by dynamical quarks, one may hope to find a phase transition similarly to pure gluodynamics. Furthermore, we 
calculate the effective potential with the chiral condensate included. We shall show that the chiral symmetry is restored in all $n$-ality sectors. This is in disagreement with the GCE description of full QCD [7, 8] and is another argument in favour of our approach. First, we review our general strategy and then apply it to $(2+1)$-dimensional QCD with Wilson fermions. Further, we use our approach to study the behaviour of chiral symmetry within the $\mathrm{CE}$ in the scheme with staggered fermions.

Let us consider the effective model of the Polyakov loops (PL) $W_{\vec{x}}$ in the strong coupling region calculated first in Ref. [9]:

$$
\int d U_{n}(x) e^{-S_{G}} \approx e^{\gamma \sum_{\vec{x}, n} S p W_{\vec{x}} S p W_{\vec{x}+n}}
$$

where $S_{G}$ is the pure gluonic action and $\gamma \sim\left(\frac{1}{g^{2}}\right)^{N_{t}}$. For a system with heavy quarks one can apply the hopping parameter expansion of the fermionic determinant in the scheme with Wilson fermions

$$
Z_{q}=\operatorname{Det}\left[D_{x y}\right] \approx e^{h \sum_{\vec{x}} S p W_{\vec{x}}+O\left(h^{2}\right)},
$$

with the hopping parameter $h=\left(\frac{1}{2 d+m}\right)^{N_{t}}$. Combining (11) and (2) one gets for the effective theory of PLs in the GCE with respect to triality

$$
Z_{G C E}=\int \prod_{\vec{x}} d W_{\vec{x}} \exp \left[\gamma \sum_{\vec{x}, n} S p W_{\vec{x}} S p W_{\vec{x}+n}+h \sum_{\vec{x}} S p W_{\vec{x}}\right]
$$

It is well known that the model (3) does not demonstrate a critical behaviour at any $\gamma$ and $h$. This is a consequence of the explicit violation of the global $Z(N)$ symmetry in the GCE.

Generalizing a simple technics for describing the system of zero triality [5, 6], one obtains the corresponding partition function in the CE

$$
Z_{C E}=\frac{1}{N_{c}} \sum_{k=1}^{N_{c}} \int \prod_{\vec{x}} d L_{\vec{x}} \exp \left[\gamma \sum_{\vec{x}, n} L_{\vec{x}} L_{\vec{x}+n}+h \sum_{\vec{x}} z_{k} L_{\vec{x}}\right],
$$

where $L_{\vec{x}}=S p W_{\vec{x}}$ is the trace of the Polyakov loop matrix and $z_{k}=\exp \left[\frac{2 \pi i}{N_{c}} k\right]$ fixes the phase sector. The integration in (舟) has to be done with the invariant measure for the $S U(N)$ group. We discuss now the $S U(2)$ gauge group. A naive effective potential for the PL in the CE for $N_{c}=2$ is of the form [6]

$$
V_{e f f}=2 d \gamma L^{2}+\ln \left(1-L^{2}\right)+\frac{1}{N} \ln (\cosh N h L),
$$


where $N$ is the number of lattice sites at a given time-slice. In the thermodynamical limit we have the term $|L h|$ in (5) which breaks $Z(2)$ symmetry at any temperature. It follows that $\langle L\rangle$ always differs from zero in this approximation. One could interpret this breaking as dynamical caused by the quark sea at low temperatures and as spontaneous at high temperatures [6]. Thus, if this is the case we have two different mechanisms of breaking. To see the critical behaviour we would need an order parameter (OP) which could distinguish between these mechanisms. The PL obviously is not suitable for this goal.

The starting point of our calculations was the simple observation that at least in the zero temperature case (or in the limit of infinite coupling) the naive mean-field does not lead to the expected result: $Z\left(N_{c}\right)$ should not be broken and $\mathrm{CE}$ and GCE descriptions have to coincide since in fact we have no ensemble at zero temperature but only a system in its ground state: The expectation value of the PL at zero temperature equals zero, whereas the naive effective potential as well as the GCE predicts $Z\left(N_{c}\right)$ is broken and the PL differs from zero in this limit.

In this respect the essential point is:

In different phase sectors in (4) the minimum of the effective action is reached for different $Z\left(N_{c}\right)$ configurations. This is very important in the disordered confinement phase where the PL may take any value. When we consider the different phase sectors on the same configurations it follows that only one of them will survive in the thermodynamical limit. However, if these configurations are indeed disordered, averaging over them should give the opposite result: reaching equal minima but for different configurations, two sectors contribute to the thermodynamical limit in the confinement phase, cancelling all noninvariant contributions.

Hence, the main idea is to execute the summation over $Z(N)$ configurations contained in the PL and only then to apply the mean field approximation. Doing in this way we can reproduce the true minima of the effective potential in each triality sector. To accomplish this one uses the formula

$$
\int d U G(U)=\frac{1}{N_{c}} \sum_{z} \int d U G(z U), z \in Z\left(N_{c}\right),
$$

which is valid for any compact group and its central subgroup $Z\left(N_{c}\right)$. This formula gives us a chance to verify whether the final result depends explicitly on the $Z\left(N_{c}\right)$ configurations contained in the initial action $S_{G}$ or not. If yes, 
we have the $Z\left(N_{c}\right)$ symmetry broken at all temperatures. In the other case, we can find a spontaneous breaking only at high temperatures.

Thus, we start from the following general expression for the EP applying Eq.(6)

$$
\begin{array}{r}
e^{N\left[V_{e f f}(L)-V(T=0)\right]}=\frac{1}{N_{c}} \sum_{k=1}^{N_{c}} \sum_{\left\{s_{x}\right\}, s_{x} \in Z\left(N_{c}\right)} \int D \mu\left(L_{x}\right) \exp \left[S_{e f f}\left(z_{k} s_{x} L_{x}\right)\right] \\
\delta\left[\sum_{x} s_{x} L_{x}-N L\right],
\end{array}
$$

where we 0 the contribution to $V_{\text {eff }}$ at zero temperature. We choose the gauge $U_{0}(x, t)=1$ for $t \in\left\{1,2, . ., N_{t}-1\right\}$ and $U_{0}\left(x, N_{t}\right)=W_{x}$ with diagonal $W_{x}$.

The effective action $S_{\text {eff }}$ in (7) is defined as an integral over all space components of gauge fields with the Wilson fermionic determinant included. Its calculation is clearly far from our present ability. Using the strong coupling and the hopping parameter expansions as well as the mean field approximation one arrives to the following effective potential in the $\mathrm{CE}$

$$
\begin{array}{r}
\exp \left[N V_{e f f}^{C E}\right]=\frac{1}{N_{c}} \sum_{k=1}^{N_{c}} \sum_{\left\{s_{x}\right\}, s_{x} \in Z\left(N_{c}\right)} \\
\exp \left[\gamma L^{2} \sum_{x, n} R e s_{x} s_{x+n}^{\star}+h L \sum_{x} z_{k} R e s_{x}+V_{I M}\right],
\end{array}
$$

with $\gamma$ and $h$ defined in (四) and (2), respectively. $V_{I M}$ stays for the invariant measure for the $S U(N)$ group. The summation over the $N_{c}$ n-ality sectors guarantees that only states contribute to the effective potential whose number of spins $s_{x}$ is a multiple of $N_{c}$.

Eqs.(7)-(8) are still valid in arbitrary dimensions and for any $S U(N)$ group. To make some further progress we consider in the following the $S U(2)$ gauge theory in $d=2+1$ dimensions. Then, our effective model (8) is two dimensional. We have to solve the $2-d$ Ising model with an effective coupling in an external field and to investigate the effective potential obtained.

After some calculations we find the following bound for the EP

$$
\begin{array}{r}
V_{e f f}^{C E}(L) \leq V_{I M}(L)+\frac{1}{N} \ln Z_{I}\left(\gamma_{L}\right)+\ln \cosh L h+ \\
\frac{1}{N} \ln \frac{1}{N} \sum_{r \neq 0} \cosh \left[N \tanh L h(<s(0) s(r)>)^{1 / 2}\right] .
\end{array}
$$


$Z_{I}$ in (9) is the partition function of the $2-d$ Ising model without external magnetic field calculated at the effective coupling $\gamma_{L}=\gamma L^{2}$.

We used the following inequality to calculate the sum over pair correlation functions

$$
\Gamma=\sum_{x_{1} \neq x_{2} \neq \ldots \neq x_{2 k}}<\prod_{i=1}^{2 k} s\left(x_{i}\right)>\leq N^{2 k-1} \sum_{r \neq 0}<s(0) s(r)>^{k} .
$$

The EP in (9) exhibits a very essential feature of the CE description, namely all odd correlation functions are identically cancelled due to the global summation over the center elements $z_{k}$. Just these contributions lead to the explicit violation of the $Z(2)$ symmetry in the GCE description. It should be stressed that the cancellation happens exactly and is not a consequence of the approximations used.

To make the following arguments as transparent as possible and to promote the analytical evaluations to the very end we use simple asymptotics for the $2-d$ Ising model with an external field at small and large values of the effective coupling $\gamma_{L}$ 10. This leads up to a constant independent of $L$ to asymptotic expressions for the EP of the form

$$
\begin{aligned}
V_{e f f}^{C E}\left(L, \gamma_{L} \ll 1\right)= & \ln \left(1-L^{2}\right)+\ln \cosh L h+2 \gamma L^{2}(\tanh L h)^{2}+ \\
& \gamma^{2} L^{4}\left[1+6(\tanh L h)^{2}-7(\tanh L h)^{4}\right]+O\left(\gamma^{3}\right)
\end{aligned}
$$

and

$$
V_{e f f}^{C E}\left(L, \gamma_{L} \gg 1\right)=\ln \left(1-L^{2}\right)+F(h) .
$$

The function $F(h)$ is defined as

$$
\begin{aligned}
& F(h)=2 \gamma L^{2}+X h+u^{2} \exp (-2 X h)+2 u^{3} \exp (-4 X h)+ \\
& u^{4}[-\exp (-4 X h)+6 \exp (-6 X h)+\exp (-8 X h)]+O\left(u^{5}\right),
\end{aligned}
$$

where $u=\exp \left(-4 \gamma L^{2}\right)$ and $X=|L|$.

Analyzing the EP (11) one concludes that at sufficiently small $\gamma$ and $h$ the maximum of the EP is located at $L=0$. As $\gamma$ and/or $h$ increases two maxima with $L= \pm\left|L_{\max }\right| \neq 0$ appear dominating the EP. In this approximation it is easy to find a critical line in the $(\gamma-h)$-plane. We omit

\footnotetext{
${ }^{2}$ In our terms the effective potential presented in [6] would correspond to $\Gamma \leq N^{2 k}$. It is obvious, however, that the bound 110 is lower.
} 
this simple calculation as further we study in details the more interesting case with the quark condensate included. The EP for $\gamma_{L} \gg 1$ (12) is also formally symmetric under $Z(2)$ transformations as it should be for the CE at any temperature. However, because of the presence of the term $X h$ in (13) the EP develops two maxima at $L \neq 0$ for any $h$. Thus, $Z(2)$ symmetry can be spontaneously broken at high temperatures indicating the deconfinement phase transition. Since we have two degenerate maxima for $\gamma \gg 1$, domain walls might separate regions with positive and negative values of $L$, similar to pure gluodynamics. Concerning this question see, however, [11. It is worth to mention that the form of the EP at large $\gamma_{L}$ is close both to the EP in the GCE and to the EP in the CE calculated in [6]. This means that the EP from [6] is only valid in the deconfinement phase.

Now, we would like to construct and to study an effective potential for Polyakov loops and for the quark condensate $\sigma$ in the CE. It has been argued in [7] that the chiral symmetry is restored only in one n-ality sector (e.g., in the real phase for $S U(3))$ while it is still broken in other sectors above the critical temperature where the chiral condensate becomes zero in the real phase. Moreover, in the phase $\arg L=\pi$ of $S U(2)$ gauge theory the chiral condensate is 1 for all temperatures [8]. We shall show that this may only be valid in the GCE. Treating QCD within the CE leads to chiral symmetry restoration in all triality sectors.

In the limit of vanishing quark mass the action for staggered fermions is chiral symmetric. Therefore, we turn now to the scheme with staggered fermions. For the effective action which includes effects of dynamical quarks in terms of mesonic field $\bar{\Psi}_{x} \Psi_{x}=\sigma_{x}$ we use the effective action of Ref.[12]. 0 this action to the $\mathrm{CE}$, we get

$$
\begin{array}{r}
\exp \left[N V_{\text {eff }}^{C E}(L, \sigma)\right]=\frac{1}{2} \sum_{k=1}^{2} \sum_{\left\{s_{x}\right\}, s_{x} \in Z(N)} \int_{-\infty}^{\infty} \prod_{x} d \sigma_{x} \exp \left[\gamma L^{2} \sum_{x, n} s_{x} s_{x+n}+V_{I M}\right] \\
\exp \left[-N_{t} \sum_{x, y} \sigma_{x} V_{x y}^{-1} \sigma_{y}\right] \prod_{x}\left[\cosh N_{t} E+z_{k} L s_{x}\right] \prod_{x} \delta\left[\sum_{x} \sigma_{x}-N \sigma\right],
\end{array}
$$

where (for $d=2$ )

$$
\sinh E=m+\sigma_{x}
$$

and

$$
V_{x y}=\frac{1}{4} \sum_{n=1}^{d}\left(\delta_{y, x+n}+\delta_{y, x-n}\right) .
$$


To get a relatively simple form for the EP we use the simplest mean-field approximation for mesonic fields $\sigma_{x}$ neglecting all fluctuations. Taking again the asymptotics for the two dimensional Ising model as described above we end up with the following expressions for the EP

$$
\begin{array}{r}
V_{e f f}^{C E}(L, \sigma)=\ln \left(1-L^{2}\right)+\ln H-N_{t} \sigma^{2}+\ln \cosh (L / H)+ \\
2 \gamma L^{2}(\tanh L / H)^{2}+\gamma^{2} L^{4}\left[1+6(\tanh L / H)^{2}-7(\tanh L / H)^{4}\right]+O\left(\gamma^{3}\right)
\end{array}
$$

for $\gamma L^{2} \ll 1$ and

$$
V_{e f f}^{C E}(L, \sigma)=\ln \left(1-L^{2}\right)+\ln H-N_{t} \sigma^{2}+2 \gamma L^{2}+F_{c}(H)
$$

for $\gamma L^{2} \gg 1$. The function $F_{c}(H)$ is of the form

$$
\begin{array}{r}
F(H)=X / H+u^{2} \exp (-2 X / H)+2 u^{3} \exp (-4 X / H)+ \\
u^{4}[-\exp (-4 X / H)+6 \exp (-6 X / H)+\exp (-8 X / H)]+O\left(u^{5}\right),
\end{array}
$$

where functions $u$ and $X$ were defined after Eq.(13). We have denoted here

$$
H=\cosh N_{t} E \text {. }
$$

These formulae are valid in the region $X / H \ll 1$. In what follows we consider $\gamma$ and $N_{t}$ as independent variables and study EPs for different values of $\gamma$ and $N_{t}$. First of all, we observe that the EP in (15) for sufficiently small $\gamma$ has the maximum at $L=0$. The EP in (16) exhibits $Z(2)$ symmetry breaking in the proper region of the convergence of the series in (17) although, similar to the EP in (12), it is also center symmetric. Hence, with respect to $Z(2)$ symmetry the behaviour of the system is close to the previous case with Wilson fermions.

We suppose now that the EP (15) reflects all qualitative features of the theory in the region $\gamma \leq 1$. This may be the case in the vicinity of $L \approx 0$ since the real expansion parameter is $\gamma L^{2}$ and therefore the series in Eq. (15) can still converge. To study the chiral symmetry behaviour we have plotted the EP (15) for several values of $N_{t}$ and $\gamma$ taking the quark mass $m=0$. Fig. 11 shows the EP at $\gamma=0.1$ for $N_{t}=10$ and for $N_{t}=4$. Because these graphics are obviously symmetric with respect to $Z(2)$ transformations we plotted them for positive $L$ only. They demonstrate that the maximum of the EP is located at $L=0$. One sees that at large $N_{t}$ the chiral symmetry is 
strongly broken $(\sigma \neq 0)$. As $N_{t}$ decreases (temperature increases) the chiral symmetry tends to restore and $\sigma \rightarrow 0$. Fig. 目 presents the EP (15) in the vicinity of the critical line for $N_{t}=2$ and $\gamma=0.8$. One observes the main maximum at $L \neq 0$ and $\sigma=0$ for these values of the parameters.

In all cases for $N_{t} \in[1-6]$ and $\gamma \in[0.5-0.9]$ the spontaneous breaking of $Z(2)$ symmetry is accompanied by chiral symmetry restoration since $\sigma=0$ above the critical point. The phase transition is of first order. The important fact is that $\sigma=0$ in both sectors, i.e. for positive and negative values of the PL. Therefore, the chiral symmetry is restored in both sectors. This is in contradiction with the GCE description where only one phase exhibits such a restoration [7, 8]. As $\gamma$ grows, the symmetric maxima of the EP are smoothly moving from each other to the values $L= \pm 1$ for the PL. In our approximation it is impossible, however to establish reliably whether they can reach these values because in this region we are far away from the strong coupling where our EP can be trusted. We have shown this behaviour in Figs. 35 plotting contour graphics for $N_{t}=3$. Fig. 3 presents the confinement region with the only maximum at $L=0$. Around $\gamma \approx 0.6$ two symmetric deconfining maxima with $\sigma=0$ start to develop. Fig. 目 shows the critical point $\gamma=0.68$ where confining and deconfining maxima become degenerate. Fig. 5 shows the deconfinement phase where the maximum with $L=0$ is metastable. There exists a rather wide region in the $\left(N_{t}, \gamma\right)$-plane where we find the coexistence of the confining vacuum with $\sigma \neq 0$ and the two degenerate deconfining vacua with $\sigma=0$. One of them is always metastable but physically acceptable because they have normal physical features in contrary to the so-called $Z(N)$ metastable phase. This picture implies the existence of different "confining" and "deconfining" bubbles around the critical line with nonzero surface tension. One could interpret this vacuum structure as an indication of a mixed state in hot nuclear matter which has been discussed for more than ten years as a hyphotetical state in the phenomenology of the quark-gluon plasma but the existence of which, to our knowledge, has not been confirmed up to now from first principles.

Let us briefly summarize our results and discuss some open questions.

In this letter we gave a prescription for the calculation of the EP in the $\mathrm{CE}$ with respect to triality. We 1 that all our results are valid in $d=3+1$ dimensions for the $S U(3)$ gauge group though the analytical calculations in this case are more difficult. The EP in the $\mathrm{CE}$ demonstrates reacher phase structure in contrast to the corresponding potential in the GCE. Three 
main points distinguish our approach from the conventional one: 1) The deconfinement phase transition manifests itself in the CE similarly to pure gluodynamics. 2) The chiral symmetry is restored together with spontaneous breaking of $Z\left(N_{c}\right)$ symmetry and this restoration takes place in all $n$-ality sectors. 3) A reach vacuum structure around the critical line indicates the existence of a mixed state in hot nuclear matter.

We showed these properties both for Wilson and for staggered fermions. The CE exhibits closer interrelation between deconfinement and chiral symmetry restoration. This connection might be even tighter if we had not used the approximation $L / H \ll 1$. It could be very instructive in this respect to include a nonzero baryonic number in the present approach as well as a finite value for the quark mass.

It has been argued in [5, 13] that $Z\left(N_{c}\right)$ global symmetry at finite temperature has a physical interpretation in terms of the charge of physical states. In our case this is the triality charge of fundamental quarks. The following question can be posed at this point: is the $Z(2)$ symmetry really broken and $L \neq 0$ above the critical point or does the presented picture give only a formal way to discover a critical behaviour? The inconsistency may arise, since if the $Z\left(N_{c}\right)$ symmetry is broken, there could be non-zero triality states contributing to the partition function [13]. It would then intuitively seem that the projection onto zero triality states and the spontaneous breakdown of $Z\left(N_{c}\right)$ symmetry are in contradiction with each other. The possible answer might be that the $\mathrm{CE}$ with respect to triality and projection of the physical observables onto zero triality states are different things: in the former case, if $Z\left(N_{c}\right)$ is broken, there are nonzero triality contributions to the free energy at high temperatures, whereas in the later case such contributions are absent. However, we tend to the opinion that in the case of the $\mathrm{CE}$ description of finite temperature QCD non-zero triality contributions are all vanishing in the thermodynamical limit. That such a vanishing is not in contradiction with the Debye screening in the deconfinement phase has been discussed in [4]. An interesting approach is to consider the Hamiltonian formulation and to interpret all physics exclusively in terms of physical space variables [11]. In this respect we reckon, that the application of the consideration of Ref. 111 to the theory with dynamical fermions described by the CE with respect to triality would give interesting results concerning critical behaviour of full QCD. We do not, however, think that $Z\left(N_{c}\right)$ symmetry is unphysical as has been discussed in [11] and 114. It is really so, that $Z\left(N_{c}\right)$ symmetry 
acts as an identity on all physical states. The reason for this is $Z\left(N_{c}\right)$ global transformations of zero temperature QCD together with the substitution

$$
\Psi(x, \beta) \rightarrow \exp \left(i \frac{2 \pi}{N} k\right) \Psi(x, 0)
$$

for the fermionic fields $\Psi$ determines the triality of quark states at finite temperature [5]. In this sense, the $Z\left(N_{c}\right)$ symmetry is a physical symmetry (i.e., if triality charges are physical charges). One has to be 0 , nevertheless, treating this symmetry at finite temperature since it may occur that not only states having zero triality charge contribute to the partition function. The CE ensures that only states contribute to the partition function which transform identically under above transformations.

\section{References}

[1] C. DeTar, Nucl.Phys. B (Proc.Suppl.) 42 (1995) 73.

[2] L. D. McLerran and B. Svetitsky, Phys.Rev. D24 (1981) 450; B. Svetitsky and L.G. Yaffe, Nucl.Phys. B210 (1982) 423; J. Kuti, J. Polonyi, K. Szlachanyi, Phys.Lett. 98B (1981) 199.

[3] A. Smilga, Report No. SUNY-NTG-95-34, hep-ph/9508305.

[4] M. Faber, O. Borisenko, S. Mashkevich, G. Zinovjev, Fresh look on triality, IK-TUW-Preprint 9209401 (1992) and 9308401 (1993);

Triality in QCD, Proc. of Int. School-Seminar'93 "Hadrons and Nuclei from QCD", ed.by K.Fyjii, Y.Akaishi, B.L.Reznik;

Nucl.Phys. B (Proc. Suppl.) 42 (1995) 484.

[5] M. Faber, O. Borisenko, G. Zinovjev, Nucl.Phys. B444 (1995) 563.

[6] M. Oleszczuk and J. Polonyi, Canonical vs. grand canonical ensemble in QCD, preprint TPR 92-34, 1992.

[7] S. Chandrasekharan, N. Christ, Dirac Spectrum, Axial Anomaly and the QCD Chiral Phase Transition, hep-lat/9509095. 
[8] M.A. Stephanov, Chiral symmetry at finite $T$, the phase of the Polyakov loop and the spectrum of the Dirac operator, hep-lat/9601001.

[9] J. Polonyi, K. Szlachanyi, Phys.Lett. 110B (1982) 395.

[10] R.J. Baxter, Exactly Solved Models in Statistical Mechanics. Academic Press, London - New York - Hong Kong - Toronto, 1982.

[11] J. Kiskis, Absence of physical walls in hot gauge theories, heplat/9510029.

[12] A. Gocksch, M. Ogilvie, Phys.Rev. D31 (1985) 877.

[13] G. Grignani, G. Semenoff, P. Sodano, O. Tirkkonen, Perugia and UBC preprints (1995), hep-th/9511110 and hep-th/9512048.

[14] A. Smilga, Ann.Phys. 234 (1994) 1. 

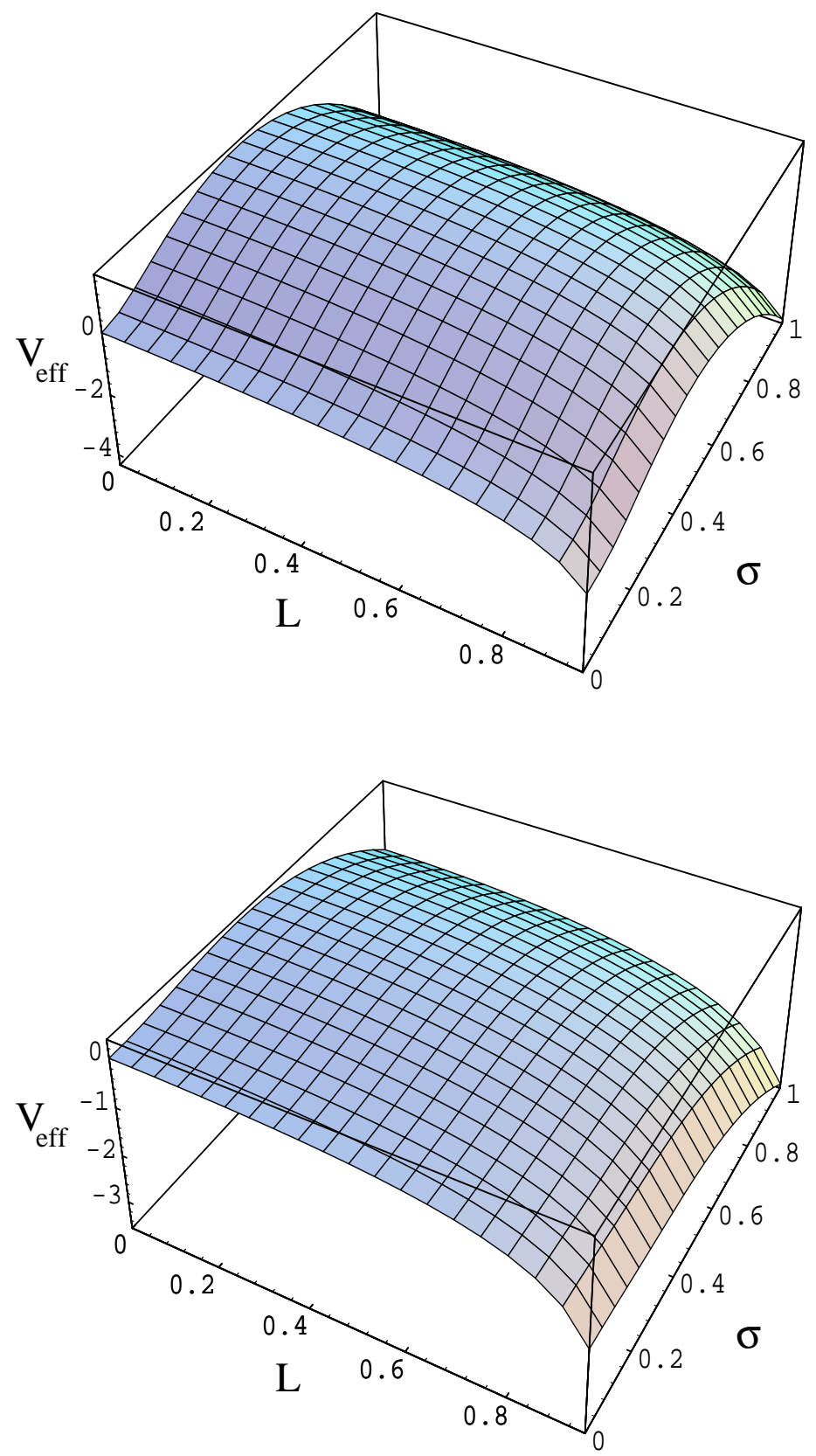

Figure 1: Effective potential in the confinement region for $\gamma=0.1, N_{t}=10$ above and $N_{t}=4$ below. 


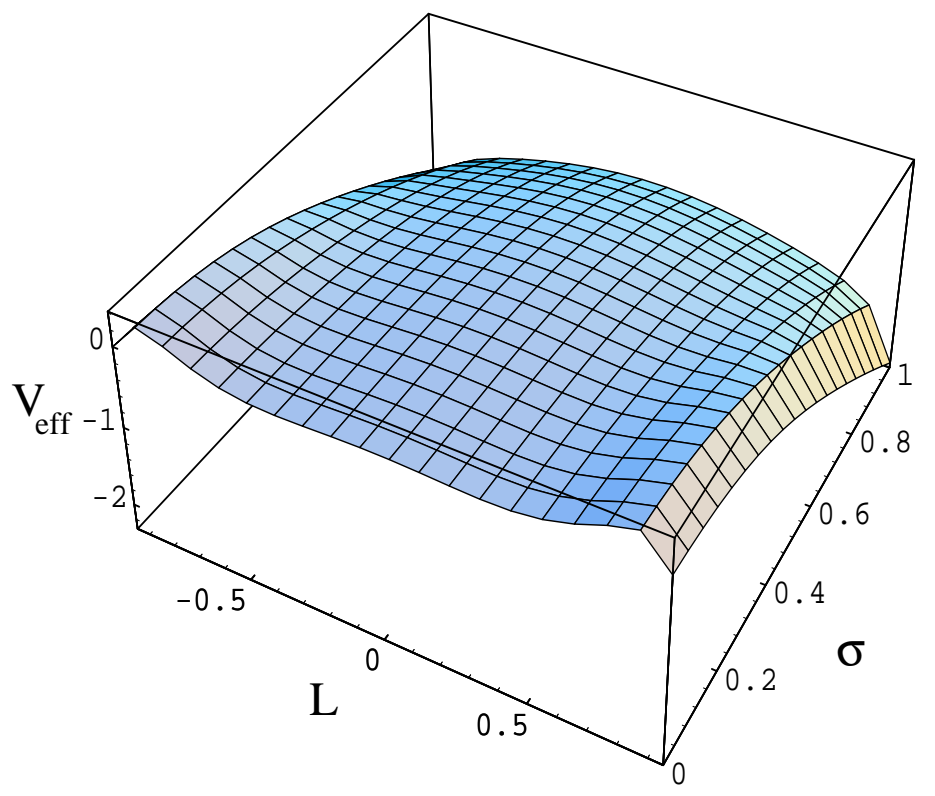

Figure 2: Effective potential around the critical line for $N_{t}=2$ and $\gamma=0.8$. 


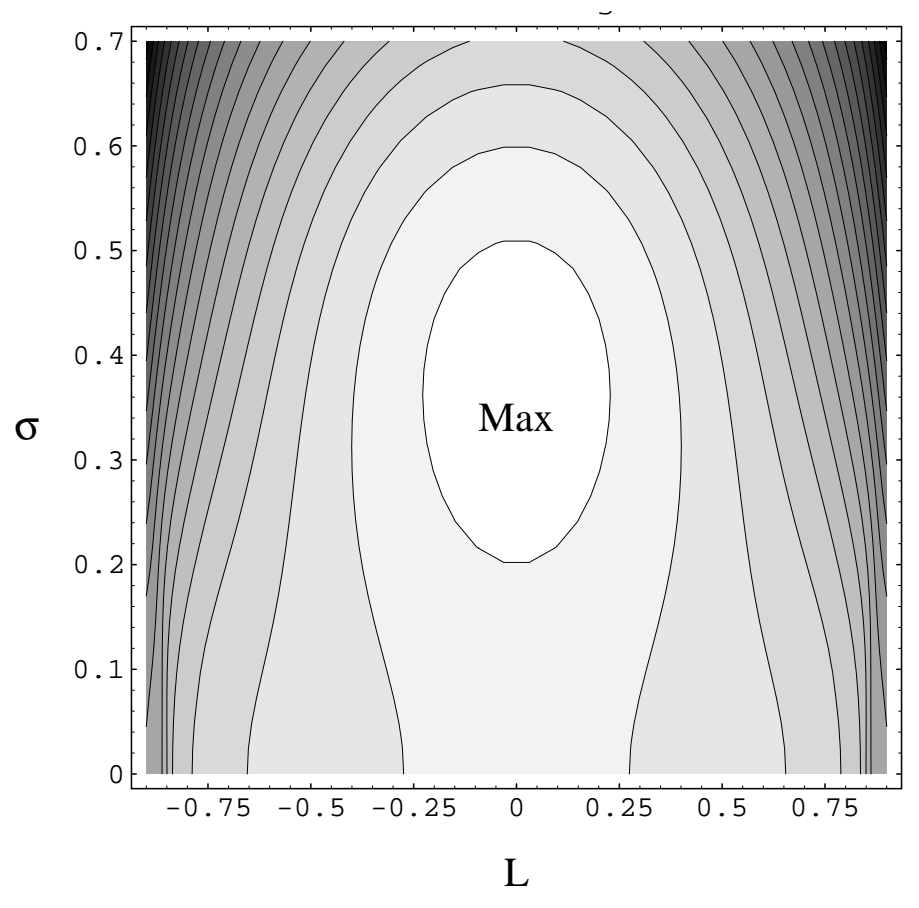

Figure 3: Contour Plot of the effective potential in the confinement region for $N_{t}=3$ and $\gamma=0.5$. 


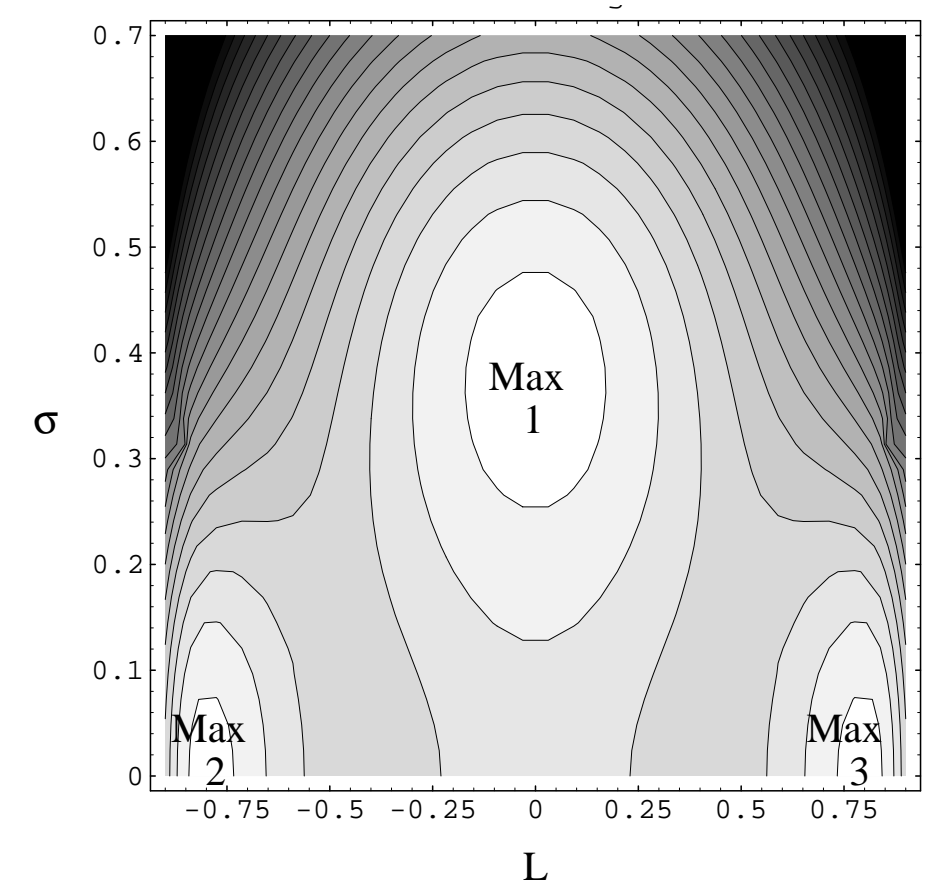

Figure 4: Contour Plot of the effective potential at the critical point $\gamma_{c}=0.68$ for $N_{t}=3$. 


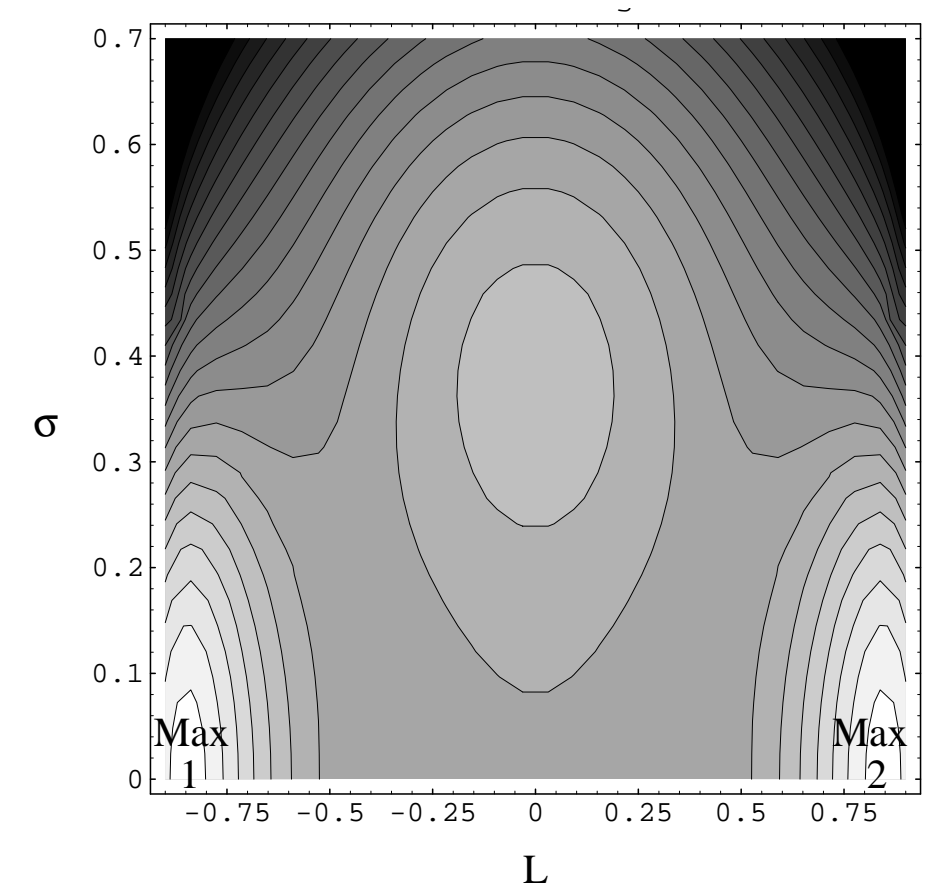

Figure 5: Contour Plot of the effective potential in the chiral symmetric phase for $N_{t}=3$ and $\gamma=0.68$. 